\title{
Reliability Analysis on Vertical Bearing Capacity of Bored Pile Determined by CPT Test
}

\author{
Chun-feng Zhao ${ }^{1,2}$, Chao $\mathrm{Xu}^{1,2}$, and Chun-mao Jiao ${ }^{1,2}$ \\ ${ }^{1}$ Key Laboratory of Geotechnical Engineering, Tongji University, Shanghai 200092, \\ China \\ ${ }^{2}$ Department of Geotechnical Engineering, School of Civil Engineering, Tongji \\ University, Shanghai 200092, China
}

\begin{abstract}
Based on the random field theory and reliability theory, the statistical methods used in the research on the probabilistic features of parameters, $P_{s}, f_{i}, P_{s b}$ had been studied. In addition, the problems with the correlation distance, pile cross section dimensions and the range of pile tip resistance, which would be used in the reliability analysis, were discussed in this paper. The reliability analyses were made on seventeen (17) construction sites based on forty-six (46) CPT test curves in Shanghai area, China. The reliability indices were obtained and the probabilistic characteristics of reliability indices were derived accordingly. The study shows that: (1) generally, the reliability index of large pile in diameter is greater than that of small pile for either prefabricated pile or cast-in-place pile, and the coefficient of variation of reliability index of prefabricated pile is less than that of cast-in-place pile; and (2) For bored piles, the variation coefficient of reliability index is less for big pile in diameter (or dimensions) than that for small pile in diameter (or dimensions)....
\end{abstract}

Keywords: Random field, Correlation distance, Reliability, CPT, Vertical bearing capacity of pile.

\section{Introduction}

Traditionally, the safety factor, $K$, is used to express whether the design of pile bearing capacity is risk or conservative. Apparently, if $K$ is larger, the design is safer, but it is not in fact. The reliability analysis with consideration of the variation of variables has become one of the hot points in the civil engineering area in the past twenty years. The researchers in the geotechinical engineering community are still facing challenges to apply the reliability analysis in their design since the nature of the variety of the soil and rock properties is difficult to recognize and the failure mode for geotechnical engineering objects is much vague. Nevertheless, the endeavor is continuing internationally. Many scholars in China have done a lot of research work to find a way to transit the traditional foundation design code, which is based on the deterministic design method, to a new foundation design code based on the reliability analysis. In this paper,the 
authors will present such research results with the vertical pile bearing capacity for prefabricated piles and the bored piles.

\section{Formulae for Determining Limit Bearing Capacity of Pile}

As the scenario of bored pile is much complex and it is still a technical challenge to make clear the borehole cross section dimensions, the slurry thickness and their functions influencing the bearing capacity of bored pile, seldom foundation design codes and guidelines recommend the formulae to determine the limit bearing capacity of bored pile by means of CPT. However, the extensive usage of bored pile in many area around the world and the CPT technology is widely used to estimate the soil engineering properties, it is necessary to explore the possibility to evaluate the bearing capacity for bored pile using the CPT results. Some scholars including the authors have done limited researches in this area (Zhao etc., 1999; Chen etc., 1994). The formula adopted for studying the reliability of evaluating the bearing capacity of bored pile by means of the specific penetration resistance of CPT is apparently same with equation (1), but the methods for evaluating the variables involved in equation (2) are different, i.e.,

$$
Q_{u}=\sum_{i=1}^{n} f_{i} U_{i} l_{i}+a_{b} P_{s b} A_{p}
$$

Where $Q_{u}$ is the bearing capacity of single pile $(\mathrm{kN}), f_{i}$ is the standard value of ultimate friction resistance for the $i$-th soil layer by means of the specific penetration resistance of $\mathrm{CPT}(\mathrm{kPa}) ; U_{i}$ and $l_{i}$ represent the average perimeter of pile and the thickness for the $i$-th soil layer respectively; $a_{b}$ is the modified coefficient for pile end resistance; $P_{s b}$ is the average specific penetration resistance of CPT near the pile end $(\mathrm{kPa}) ; A_{p}$ is the cross section area of pile end $(\mathrm{m} 2) ; n$ is the number of soil layer that pile penetrates.

\section{Calculation Methods of the Probabilistic Features of the Specific Penetration Resistance $\boldsymbol{P}_{s}$}

For the standard values of the ultimate friction resistance and ultimate end resistance are evaluated based on the specific penetration resistance $P_{s}$ of CPT according to empirical formulae, their probabilistic characteristics will depend on the statistical methods, i.e., how to get the parameter of $P_{s}$. The $P_{s}$ value of CPT varies continuously along with the penetration depth, so the question that how the $P_{s}$ value is calculated based on the CPT test curve for a foundation soil layer arises. As there is autocorrelation of soil parameters, and the independence of choosing individual $P_{s}$ value should be guaranteed. The probabilistic properties (variance and variation coefficient) of $P_{s}$ value should be evaluated taking the correlation distance of typical soil layer in shanghai area into consideration. 
The calculated variance is so called 'point variance', and the variation coefficient based on point variance can be gotten. Then authors obtain the variation coefficient for a soil layer based on one-dimensional random field theory (Vanmarcke, 1977), i.e.,

$$
V_{i}=V_{i}^{\prime} \sqrt{\frac{\delta_{i, 0}}{l_{i}}}
$$

Where $V_{i}^{\prime}$ represents the variation coefficient derived from the point variance for the $i$-th soil layer of a CPT hole, $V_{i}$ is the variation coefficient derived from one-dimensional random field theory for the $i$-th soil layer of the CPT hole, $\delta_{i, 0}$ is the correlation distance for the $i$-th soil layer, $l_{i}$ refers to the thickness for the $i$-th soil layer.

Preceding discussion only considers the soil parameter variation along depth; the variability in horizontal direction also needs to be taken into account. For there are only a few CPT holes and the distances between holes of CPT are relatively large in a construction site, the random variable theory was adopted to calculate spatial average variance of a site approximately. The spatial variation coefficient $V_{h i}$ is evaluated by Equation (3):

$$
V_{i}=V_{h}^{\prime} \frac{1}{\sqrt{m}}
$$

Where $V_{h}^{\prime}$ is the variation coefficient for a site, which is calculated based on the point variance; $m$ is the number of the hole of CPT.

\subsection{The Method for Evaluating $\boldsymbol{P}_{s}$ in a Site}

While calculating the statistical properties of the CPT specific resistance, $P_{s}$, by means of random field theory, very probably, the thickness of soil layer cannot be divided by the correlation distance neatly. For solving the question, the practical thickness is divided by correlation distance, then round off to the nearest whole number, the real thickness of soil layer is replaced by the multiplication of the correlation distance and the round number. As shown in Fig.1, where $h_{i l}^{\prime}$ is thickness of the CPT hole for the $i$-th soil layer, $h_{i l}$ is the soil layer thickness used in the calculation. Here $i$ represents the serial number of soil layer, $i=1, \cdots, n$; $l$ refers to the serial number of CPT hole in one site, $l=1, \cdots, m$. The process of evaluating the statistical properties of $P_{s}$ is as followed.

(1) Rounding $\left[\frac{h_{i l}^{\prime}}{\delta_{i, 0}}\right]$ to a nearest whole number, $N$, then replacing the real soil layer thickness by $h_{i l}=N \cdot \delta_{i, 0}$.

(2) Averaging $P_{s}$ in the range of $h_{i l}$ to obtain $\bar{P}_{\text {sil }}$, calculating the standard point variance $\sigma_{\text {sil }}^{\prime}$ and the point variation coefficient $V_{s i l}^{\prime}$ of $P_{s}$.

(3) Calculating the average standard variance $\sigma_{\text {sil }}$ and average variation coefficient $V_{\text {sil }}$ of $P_{\text {sil }}$ in the range of $h_{i l}$, i.e.,

$$
\sigma_{s i l}=\sigma_{s i l} \sqrt{\frac{\delta_{i, 0}}{h_{i l}}}, \quad V_{S i l}=V_{S i l} \sqrt{\frac{\delta_{i, 0}}{h_{i l}}}
$$




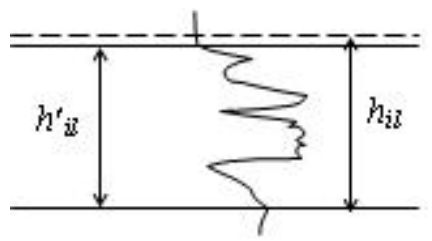

Fig. 1. The profile for $i$-th soil layer in $l$-th CPT hole

(4) Averaging the variation coefficient $V_{\text {sil }}$ of the $i$ th soil layer along the depth for a site, i.e.,

$$
\bar{V}_{s i}=\frac{1}{m} \sum_{i=1}^{n} V_{s i l}
$$

(5) Averaging the mean value of the specific resistance, $\bar{P}_{\text {sil }}$ of the $i$-th soil layer for a site and calculating the field variation coefficient $V_{h i}$ across the site, i.e.

$$
\bar{P}_{s i}=\frac{1}{m} \sum_{i=1}^{m} \bar{P}_{s i l} \quad V_{h i}=\frac{\left[\frac{1}{m-1} \sum_{i=1}^{m}\left(\bar{P}_{s i l}-\bar{P}_{s i}\right)^{2}\right]^{\frac{1}{2}}}{\bar{P}_{s i}}
$$

(6) Calculating the spatial variation coefficient $V_{i}$ for the $i$-th soil layer and the average standard variance $\sigma_{P_{s i}}$, i.e.,

$$
V_{i}=\left(\bar{V}_{S i}^{2}+\frac{1}{m} V_{h i}^{2}\right)^{\frac{1}{2}}, \quad \sigma_{P_{s i}}=\bar{P}_{s i} \cdot V_{i}
$$

\section{The Methods for Evaluating the Friction Resistance $f_{i}$}

In the case of bored pile, as the pile diameter is influenced by several factors, such as the soil properties, the construction process and quality, it is still a difficult task in the civil engineering community to get the statistical properties of the diameter of bored pile. One thing is clear, whether for a single soil layer or for the total soil layers along the pile shaft, the shaft friction resistance calculated according to the estimated pile diameter should be equivalent to the practical friction resistance. The author has made attempt to deal with the problem (Zhao, 1998). In this paper, the diameter of the bored pile is assumed as the design diameter for lacking test data. For the bored pile, the side friction resistance $f_{i}$ of the $i$-th soil layer can be written as,

$$
f_{i}=a+b P_{s i}+c P_{s i}^{2}
$$

Where $a, b, c$ are coefficients, which can be evaluated based on the soil properties.

(1) For shallow topsoil (the depth is not beyond 6m),

$$
a=15 k P a \quad b=c=0
$$


(2) For clay, whose average specific resistance satisfies $\overline{P_{s l}} \leq 5000 \mathrm{kPa}$,

$$
a=8.775 k P a \quad b=0.029 \quad c=-2.551 \times 10^{-6}
$$

(3) For clay, whose average specific resistance satisfies $\bar{P}_{s l} \geq 5000 \mathrm{kPa}$

$$
a=90 k P a \quad b=c=0
$$

(4) For clay $\left(\bar{P}_{s l} \geq 15000 k P a\right)$, sandy silt, and sand,

$$
a=18.705 k P a \quad b=0.010 \quad c=-3.498
$$

(5) For clay $\left(\bar{P}_{s l} \geq 000 k P a\right)$, sandy silt, and sand,

$$
a=90 k P a \quad b=c=0
$$

While bearing stratum is sand or silt and the pile end penetrates into this layer less than $10 d$ ( $d$ represents the design pile diameter), the side friction resistance in the range of $4 d$ above the pile end should be modified as follow,

$$
f_{i}^{*}=\eta f_{i}, \quad(\eta=0.5)
$$

Supposing $P_{s i}$ subject to normal distribution, the statistical properties of side friction resistance, $f_{i}$, for the bored pile can be derived based on Equation (8) and the error propagation theory, viz.,

$$
\begin{gathered}
\bar{f}_{i}=a+b \bar{P}_{s i}+c\left(\bar{P}_{s i}^{2}+\sigma_{P_{s i}}^{2}\right) \\
\sigma_{P_{s i}}^{2}\left(f_{i}\right)=b^{2} \sigma_{P_{s i}}^{2}+4 b c \bar{P}_{s i} \sigma^{2}+4 c^{2} \bar{P}_{s i}^{2} 2 \sigma_{P_{s i}}^{2}+2 c^{2} \sigma_{P_{s i}}^{4} \\
V_{f_{i}}=\frac{\sigma_{P_{s i}}\left[\left(b+2 c \bar{P}_{s i}\right)^{2}+2 c^{2} \sigma_{P_{s i}}^{2}\right]^{\frac{1}{2}}}{a+b \bar{P}_{s i}+c\left(\bar{P}_{s i}^{2}+\sigma_{P_{s i}}^{2}\right)}
\end{gathered}
$$

\section{The Methods for Evaluating the Pile Tip Resistance $P_{s b}$}

The evaluating methods of statistic property of the tip resistance $P_{s b}$ for bored pile are similar to the methods for the prefabricated pile, but the calculating range for the bored pile is different, it includes $4 \mathrm{D}$ ( $\mathrm{D}$ refers to the diameter of the bored pile) above the pile end and 1D under the pile end, as showed in fig. 2 (Chen Qianghua, etc., 1994). The methods for evaluating the statistic properties of the tip resistance $P_{s b}$ for the bored pile are briefly discussed as followed.

(1) As the same reason to consider the factor of correlation distance, and the ratios of $\frac{2 d}{\delta_{i, 0}}, \frac{4 d}{\delta_{i, 0}}$ and $\frac{6 d}{\delta_{i-1,0}}$ may not the integer(s), authors take the same strategies to round the ratios to whole numbers. After these manipulations, the integer 


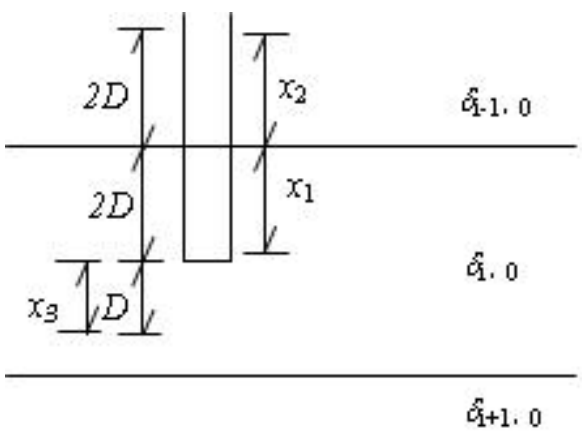

Fig. 2. The skeleton for the pile tip resistance analysis of the bored pile

parts of $\frac{2 d}{\delta_{i, 0}}, \frac{4 d}{\delta_{i, 0}}$ and $\frac{6 d}{\delta_{i-1,0}}$ are expressed by $n_{1}, n_{2}$ and $n_{3}$ and the corresponding thickness are $x_{1}, x_{2}$ and $x_{3}$, respectively. (2) The thickness weighted average specific penetration resistance, $\bar{P}_{i l 1}$ and the average variation coefficient, $V_{i l}$, in the range of $x_{1}+x_{2}$ for the l-th CPT hole are evaluated by equation (18).

$$
\begin{aligned}
V_{i l 1} & =\frac{x_{2} V_{s i-1 l 2}+x_{1} V_{s i l 1}}{x_{1}+x_{2}} \\
\bar{P}_{i l 1} & =\frac{x_{2} \bar{P}_{s i-1 l 2}+x_{1} \bar{P}_{s i l 1}}{x_{1}+x_{2}}
\end{aligned}
$$

(3) The variation coefficient, $V_{i l}$ and the average specific penetration resistance, $\bar{P}_{i l}$ in the range of $x_{1}+x_{2}$ for the $i$-th soil layer in a site are obtained as followed.

$$
V_{i l}=\frac{1}{m} \sum_{l=1}^{m} V_{i l 1}, \quad \bar{P}_{i l}=\frac{1}{m} \sum_{l=1}^{m} \bar{P}_{i l 1}
$$

(4) In the same way, we can obtain the average specific penetration resistance, $\bar{P}_{s i l 3}$, in the rang of $x_{3}$, and the average variation coefficient, $V_{s i l 3}$, for $i$-th soil layer in the $l$-th CPT hole and the variation coefficient, $V_{i 2}$, for $i$-th soil layer in a site.

$$
\begin{gathered}
V_{s i l 3}=0 \quad\left(n_{3}=1\right) \\
V_{s i l 3}=V_{s i l 3}^{\prime} \sqrt{\frac{1}{n_{3}}} \quad\left(n_{3} \geq 2\right) \\
V_{i 2}=\frac{1}{m} \sum_{l=1}^{m} V_{s i l 3}
\end{gathered}
$$

(5) The average specific penetration resistance, $\bar{P}_{i 2}$, in the range of $x_{3}$ within the territory of a site is easily obtained by equation (21).

$$
\bar{P}_{i 2}=\frac{1}{m} \sum_{l=1}^{m} P_{i l 3}
$$


(6) The field variation coefficients of above the pile end and under the pile end for the $i$-th soil layer are calculated by equations (22), respectively.

$$
V_{h i l}=\frac{\left[\frac{1}{m-1} \sum_{i=1}^{m}\left(P_{i l 1}-\bar{P}_{i 1}\right)^{2}\right]^{\frac{1}{2}}}{\bar{P}_{i 1}}, \quad V_{h i 2}=\frac{\left[\frac{1}{m-1} \sum_{i=1}^{m}\left(P_{s i l 3}-\bar{P}_{i 2}\right)^{2}\right]^{\frac{1}{2}}}{\bar{P}_{i 2}}
$$

(7) The total variation coefficient of above the pile end and under the pile end for the $i$-th soil layer are calculated by equations (23).

$$
V_{P 1}=\left(\frac{1}{m} V_{h i l}^{2}+V_{i l}^{2}\right)^{\frac{1}{2}}, \quad V_{P 2}=\left(\frac{1}{m} V_{h i 2}^{2}+V_{i 2}^{2}\right)^{\frac{1}{2}}
$$

(8) The average pile tip resistance for bored pile, $P_{s b}$, and the variation coefficient $V_{s b}$ are determined by equation (24).

$$
P_{s b}=\frac{\bar{P}_{i 1}+\bar{P}_{i 2}}{2}, \quad V_{s b}=\frac{\left(V_{P 1}^{2} \bar{P}_{i 2}^{2}+a^{2} V_{P 2}^{2} \bar{P}_{i 2}^{2}\right)^{\frac{1}{2}}}{\bar{P}_{i 1}+\bar{P}_{i 2}}
$$

\section{Reliability Analysis and the Results}

The limit state equation of the pile bearing capacity failure model for the bored pile can be written as,

$$
\sum_{i=1}^{n} f_{i} U_{i} l_{i}+a_{b} P_{s b} A_{p}-Q_{G}-Q_{L}=0
$$

This is a linear equation, which contains $n+3$ variables. That all these basic variables are subject to normal distribution is presumed herein, the reliability index, $\beta$, can be calculated on equation (37).

$$
\beta=\frac{m_{P_{s b}} a_{b} A_{P}+U_{P} \sum_{i=1}^{n} m_{f_{i}} l_{i}-m_{Q_{G}}-m_{Q_{L}}}{\left(a_{b}^{2} A_{P}^{2} \sigma_{P_{s b}}^{2}+U_{P}^{2} \sum_{i=1}^{n} \sigma_{f_{i}}^{2} l_{i}^{2}+\sigma_{Q_{G}}^{2}+\sigma_{Q_{L}}^{2}\right)^{\frac{1}{2}}}
$$

Where $m_{P_{s b}} m_{f_{i}}, m_{Q_{G}}, m_{Q_{L}}$ are the average of corresponding variables, $\sigma_{P_{s b}}, \sigma_{f_{i}}$, $\sigma_{Q_{G}}, \sigma_{Q_{L}}$ are the standard variance of corresponding variables.

The results of reliability on the bored pile in seventeen (17) construction sites in Shanghai area are presented in table 3 . In the calculation of the reliability index, $\beta$, based on JC method, the safety factor $K=2$ is assumed, the bored piles with different diameters $(650 \mathrm{~mm}$ and $800 \mathrm{~mm})$ are considered, and the different load ratios (0.2 and 0.5) are set in the computation.

It should be noted that the variation of the analysis mode and the bored pile diameter weren't taken into account in the above reliability analyses. If these factors were considered, the reliability indexes would decrease in theory. These problems definitely need further research work. 
Table 1. The statistical properties of the reliability index $\beta$ for bored piles

\begin{tabular}{ccccc}
\hline Pile diameter & \multicolumn{2}{c}{$650 \mathrm{~mm}$} & $800 \mathrm{~mm}$ & \\
\hline Load ratios & 0.2 & 0.5 & 0.2 & 0.5 \\
Average & 4.889 & 4.573 & 4.940 & 4.618 \\
Standard variance & 0.576 & 0.475 & 0.489 & 0.398 \\
Variation coefficient & 0.118 & 0.104 & 0.099 & 0.086 \\
\hline
\end{tabular}

\section{Conclusions}

(1)The reliability index is larger for big pile in diameter (or dimensions) than that for small pile in diameter (or dimensions).

(2) The variation coefficient of reliability index is less for big pile in diameter (or dimensions) than that for small pile in diameter (or dimensions).

(3) The results in tables 3 show that the reliability indexes are very large, which indicates that the method to estimate the limit bearing capacity of pile by using the specific penetration resistance based on equation (1).

\section{References}

1. Shanghai Standard: Foundation design code S DBJ08-11-1999

2. China National Code: Architecture pile foundation technology standard S JGJ 94-94

3. Zhao Chunfeng, Ye Guanbao: Reliability analysis on the bearing capacity of bored pile evaluated by CPT. Rock and Soil Mechanics, 201999 65-68

4. Cheng Qianghua:the bearing capacity of single bored pile evaluated by CPT. China architecture and building press, Proceedings of the 7th Conference on Soil Mechanics and Foundation Engineering.1994:373-376

5. Zhao Chunfeng: reliability analysis on pile foundation in Shanghai region, the thesis for degree of doctor of Tongji University, 1998 96-99

6. Vanmarcke, E.H.: Probabilistic modeling of soil profile. ASCE, GT11,103 1977 1227-1246

7. Zhao Chunfeng: Research on partial factors of recommended limit bearing capacity formula of precast pile in Shanghai Foundation Design Code,Engineering Mechanics, 20 2003:141-144

8. Zhao Chunfeng: Research on partial factors of vertical bearing capacity of cast-inplace pile, Engineering Mechanics,23 2006:126-130 